Review

\title{
Ovarian Germline Stem Cells in the Teleost Fish, Medaka (Oryzias latipes)
}

\author{
Shuhei Nakamura ${ }^{1}$, Kayo Kobayashi ${ }^{1}$, Toshiya Nishimura ${ }^{1,2}$, and Minoru Tanaka ${ }^{1,2}$ 凶 \\ 1. Laboratory of Molecular Genetics for Reproduction, National Institute for Basic Biology, Okazaki 444-8787, Japan. \\ 2. Department of Basic Biology, Graduate University for Advanced Studies (SOKENDAI), Okazaki, Aichi 444-8585, Japan.
}

$\bowtie$ Corresponding author: FAX +81 56459 5851, Email: mtanaka@nibb.ac.jp (Minoru Tanaka)

(C) Ivyspring International Publisher. This is an open-access article distributed under the terms of the Creative Commons License (http://creativecommons.org/ licenses/by-nc-nd/3.0/). Reproduction is permitted for personal, noncommercial use, provided that the article is in whole, unmodified, and properly cited.

Received: 2011.01.01; Accepted: 2011.04.01; Published: 2011.04.14

\begin{abstract}
In the mammalian testis germline stem cells keep producing many sperms, while there is no direct evidence for the presence of germline stem cells in the ovary. It is widely accepted in mammals that the mature oocytes are supplied from a pool of primordial follicles in the adult ovary. In other vertebrates, such as fish, however, there has been no investigation on the mechanism underlying the high egg-producing ability. In this review, we introduce the recently identified ovarian germline stem cells and the surrounding unique structure in teleost fish, medaka (Oryzias latipes) [Nakamura S et al. Science. 2010; 328: 156I-1563]. We also discuss about the expression and function of sox 9 that characterizes this unique structure.
\end{abstract}

Key words: medaka, germline stem cells, sox 9

\section{Introduction}

In mammalian ovaries, it is well known that mitotic division of germ cells completes before birth. Neo-oogenesis does not occur afterwards. Therefore the number of oocytes to be ovulated is limited to the number of primordial follicles that are generated during embryogenesis. In contrast, in many vertebrates with high fecundity, the number of oocytes is thought to be infinite. In the adult ovary of these species actually, mitotic germ cells are histologically observed as oogonia and thus the proliferation of oogonia can supply mature eggs continuously throughout the life. However, the molecular and cellular mechanisms underlying the continuous production of oocytes have not been well addressed in these species. In this review, we introduce the recently identified germline stem cells in adult medaka ovary and the possible function of sox 9 gene that expresses in the ovarian niche structure [1].

\section{Oogenesis in the vertebrate ovary}

Conceptually gametogenesis has to proceed in a chronological order of oogenesis and spermatogene- sis, mitotic division to increase the number of gonial cells, meiosis to prepare a reductive set of chromosomes and a final maturing process to produce fertile eggs and sperms. But these distinctive steps of gametogenesis do not always proceed as successive events.

In most vertebrates, the adult testis contains all the steps of spermatogenesis and some population of spermatogonia function as germline stem cells, ensuring the production of tremendous number of sperm throughout the life. The composition of germ cells in the adult ovary, however, varies among vertebrates. In the mammalian ovary, all oogonia finish synchronous cystic divisions and enter subsequent meiosis by the date of delivery. During the development of ovary, a pool of primordial follicles is formed and some germ cells are eliminated by apoptosis [2]. All the primordial follicles are supplied from oogonia, in a short period of ovarian development around birth, through synchronous division and meiosis in cord-like structures extending from the cortical region (Figure 1). 

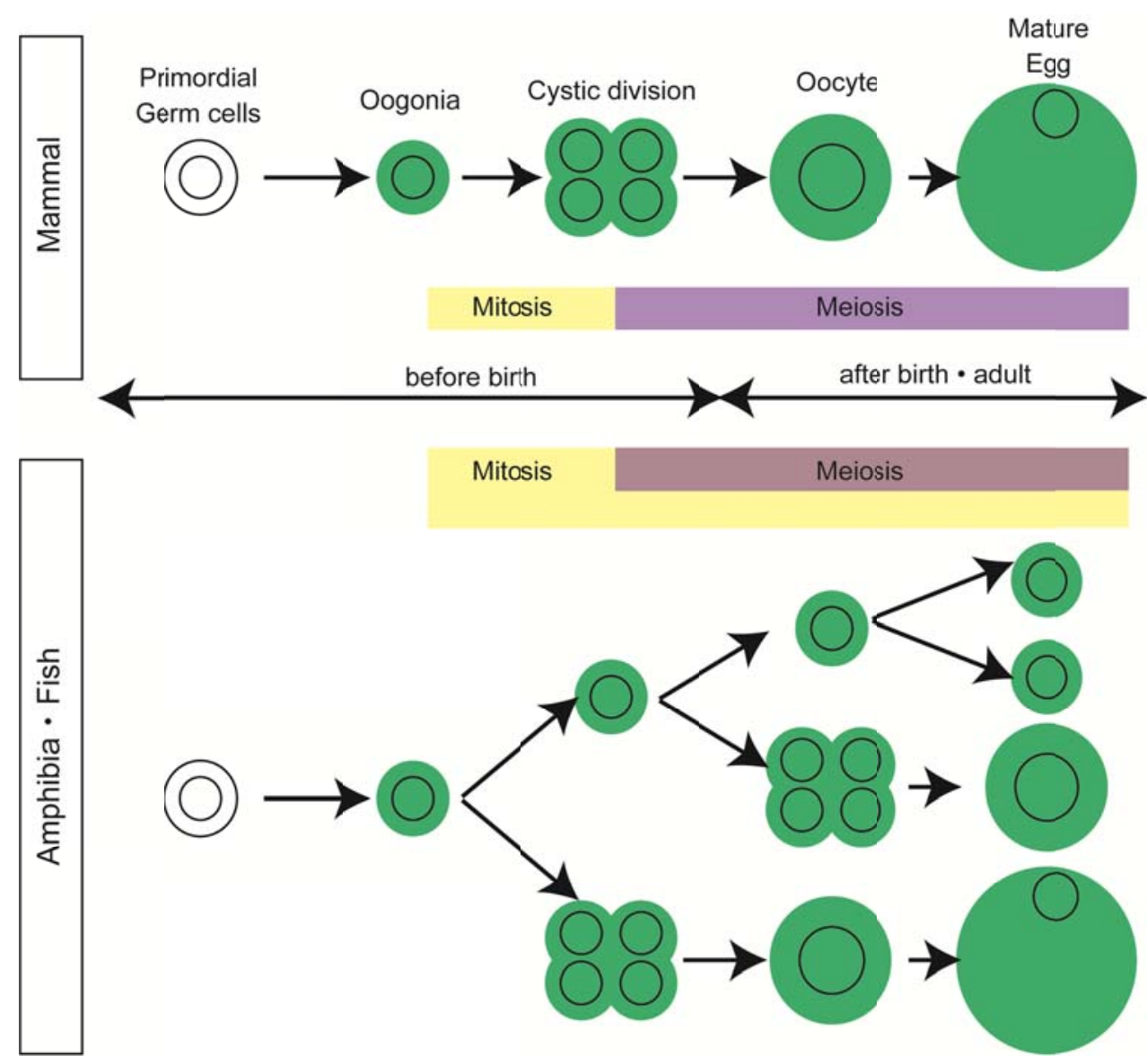

Figure I. Oogenesis in vertebrates. In mammals, mitotic division of oogonia finish before birth and meiotic germ cells are only present in postnatal and adult ovaries. In contrast, germ cells in all the stages of oogenesis are present in adult ovaries of high fecundity species, such as amphibia and fish.

Mitotic germ cells, a good indication of oogonia, are no longer observed in ovary of many mammals after birth $[2,3]$.

In contrast, in the ovary of animals with high egg-producing ability, such as fish and amphibia, mitosis in oogonia is observed histologically in adult mature ovary $[4,5]$ (Figure 1 ).

\section{Are germline stem cells present in mamma- lian ovary?}

It has been generally accepted, as mentioned above, that mammalian ovary loses mitotic oogonia after birth. On the other hand, there has been controversial by the experts of reproduction and the stem cell biologists on the presence of germline stem cells that might produce oocytes in mature ovary. In 2004, Tilly and his colleagues showed that stem cell-like germ cells are present on the surface epithelium of ovary and neo-oogenesis actually occurs in mammalian adult ovary [6]. They also showed that these germ cells expressed Mouse Vasa Homolog, MVH and a meiosis marker, SCP3. BrdU incorporation analysis indicated that these germ cells undergo mitosis. They also described that the numbers of follicle cells were decreased after the busulfan treatment, which abolishes only the mitotic cells. In addition, when the ovarian fragments expressing GFP were grafted to the wild-type ovary, GFP-positive oocytes differentiated in the host ovary. Next year, the same group showed that MVH-positive cells were present in the bone marrow and peripheral blood and bone marrow transplantation restored the oocyte production in the Atm knockout mouse, which would otherwise be incapable of making oocytes [7]. However, these result are now controversial, because the papers from Wagers' group argued that the ovulated oocytes were not derived from the bone marrow or peripheral blood in 2005 [8]. Although further experiments are required, there is no direct evidence of germline stem cells producing the functional eggs in adult mammalian ovary. 


\section{'Ovarian Cords' in medaka ovary}

Many fish and amphibia have high fecundity and exhibit a variety of reproductive strategies. Salmonid fish spawns eggs once a year while goldfish spawn many times in one reproductive season within a year. Some tropical fish has no particular spawning season and spawns many times per year. The number of eggs to be spawned varies, from a few to multibillion as seen in headfish. In the fish ovaries, mitotic oogonia are morphologically observed quite often after birth [4] and therefore, unlike mammals, new eggs are thought to be produced in mature ovary. A sort of 'germline stem cells' with mitosis, if present, could be the source of many eggs. Alternatively, certain differentiated oogonia also could provide a large pool of follicles through mitosis. But the cellular and molecular mechanisms ensuring supply of eggs from oognia have been unclear and have remained to be addressed as a question of broad interest.

We study the mechanism underlying the formation of ovary and testes using teleost fish, medaka (Oryzias latipes) as a model. Medaka spawns several dozens of eggs every day for about one year in artificial long-daylight conditions. Our laboratory has succeeded in visualizing several cell lineages constituting gonads by establishing the transgenic medaka $[9,10]$. Additionally, we have also succeeded in applying both a cre/loxP system and a heat -inducible system to medaka. By using these techniques, we have recently identified germline stem cells and the surrounding unique structure in medaka adult ovaries [1].

Medaka ovary is composed of two major compartments; the ovarian cavity on a dorsal side of the ovary, to which the mature eggs are ovulated, and the stromal compartment on a ventral side, in which the folliculogenesis and oocyte maturation proceed. (Figure 2A).

The multilayer epithelium, called 'germinal epithelium', with the basement membrane on the ventral side, lies between the two compartments. The mature eggs are ovulated from the stromal compartment through the germinal epithelium into the ovarian cavity (Figure 2A).

We established the transgenic medaka in which, medaka homolog of mammalian sox9, sox $9 b$-expressing cells are visualized [11]. It is known that mammalian sox 9 is essential for testis development and is not expressed in the developing ovary [12-15]. However, we have noticed that sox9b is ex- pressed in the medaka ovary and found that oogonia at early stages of oogenesis are all surrounded by sox $9 b$-expressing cells to form 'units'. We named the unit 'germinal cradle'. The morphological analysis revealed that the germinal cradles are connected each other by cellular processes of sox $9 b$-expressing cells to form the network. We found the network buried within the germinal epithelium, between a single layer of epithelial cells and the basement membrane (Figure $2 \mathrm{~A}$ and $\mathrm{B}$ ). We named the whole network 'ovarian cord'.

In the germinal cradles, germ cells can be categorized to three types by their morphology and the way germ cells are surrounded by sox $9 b$-expressing cells; (1) single isolated germ cells which are completely surrounded by sox $9 b$-expressing cells one by one (Gs), (2) cyst-forming germ cells which are connected by the intercellular bridge and surrounded by sox $9 b$-expressing cells as a cluster (Gcys) and (3) large oocytes in the meiotic diplotene stage which are surrounded by sox $9 b$-expressing cells one by one (Gdip) (Figure 2C). Although there are several types of germinal cradles by combination of the three types of germ cells, any of germinal cradles has one to five numbers of single isolated germ cells. We also found that some cyst-forming germ cells are composed of early stages of meiotic germ cells before diplotene stage.

\section{Identification of ovarian germline stem cells}

Previously, we found nanos 2 is expressed in both spermatogonial and oogonial cells [16]. Therefore we have checked the nanos 2 expression in the germinal cradle and have found that nanos2 is exclusively expressed in the single isolated germ cells (Gs type of germ cells). We hypothesized that germline stem cells are present in the nanos2-exressing cells and conducted the clonal analysis using the heat-inducible cre/loxP system [1]. We have generated transgenic medaka with hsp-cre:mCherry and nos $2 p$-loxP[DsRed]-EGFP-olvas3'UTR transgenes. With the design of these transgenes, the floxed region with DsRed is excised after heat treatment, which would lead to the transient transcription of EGFP only in a nanos2-expressing Gs type of germ cells. But, since the action of olvas 3'UTR fused to EGFP gene directs the translation of EGFP and confers EGFP transcripts with the increased stability in all types of germ cells $[10,17]$, the progeny of naons2-expressing cells can be kept tracked by the EGFP fluorescence. 

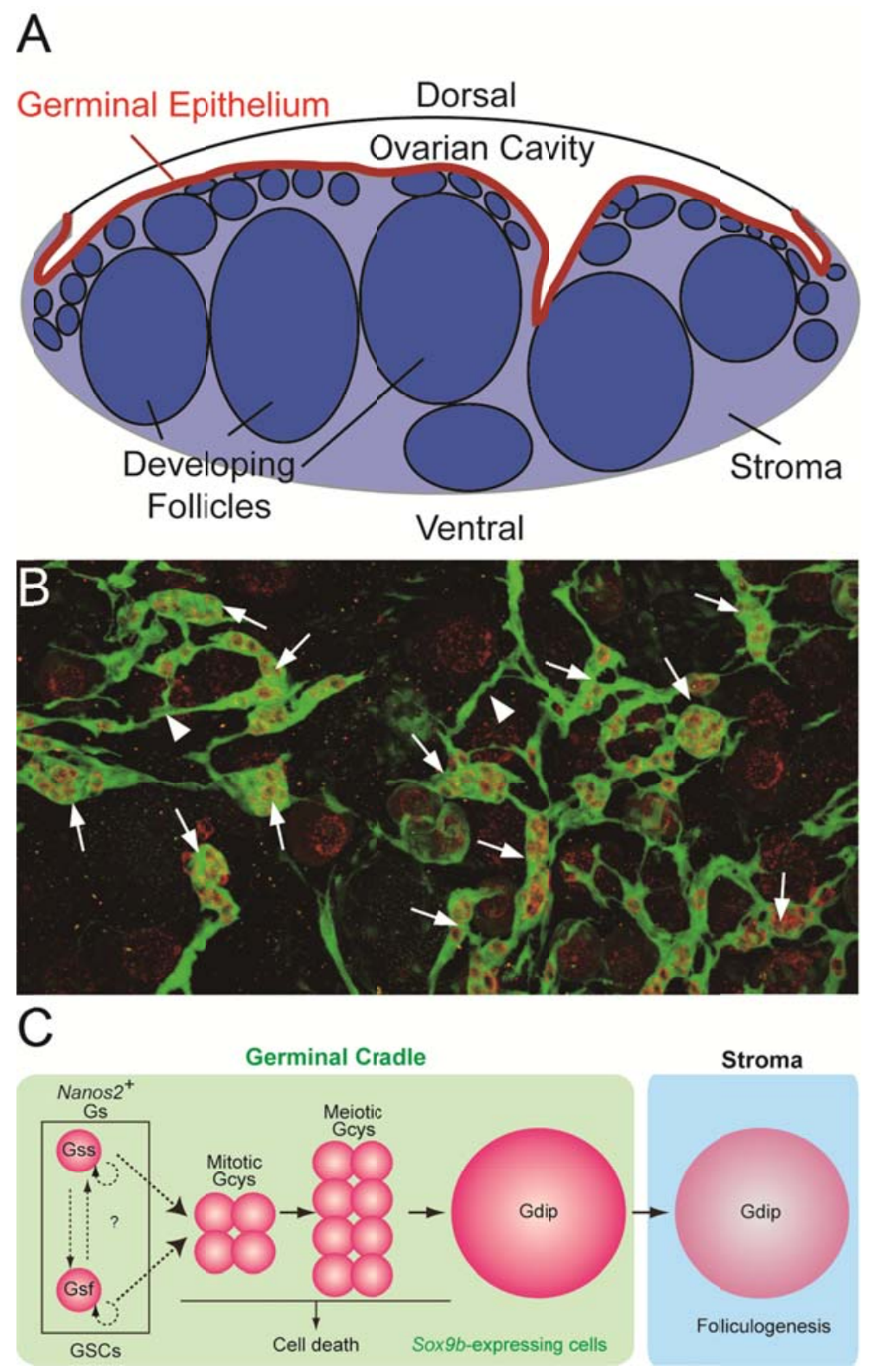

Figure 2. Ovarian cord in medaka ovary. (A) The transverse section of medaka ovary. Medaka ovary consists mainly of two compartments; the ovarian cavity to which mature oocytes are ovulated and the stroma in which folliculogenesis and oocyte maturation proceeds. Between these two compartments, there is the germinal epithelium (red) lined by the basement membrane. (B) A dorsal view of the ovarian cord in ovary of sox9b-EGFP transgenic medaka. Sox $9 b$-expressing cells are visualized with EGFP expression immunostained with anti-GFP (green) antibody. Germ cells are stained by anti-OLVAS antibody (red). 'Germinal cradles' (arrows) composed of germ cells (red) and sox $9 \mathrm{~b}$-expressing cells are connected each other by the cellular processes of sox 9 -expressing cells (arrowheads) to form the network, 'ovarian cords'. (C) The model for early oogenesis in medaka. In the germinal cradle, three types of germ cells are present; nanos2-positive single isolated germ cells (Gs), cyst-forming germ cells (Gcys) and diplotene oocytes (Gdip). Fast-dividing (Gsf) and slow-dividing (Gss) germ cells exist among Gs and both or either of them function as germline stem cells (GSCs). Gs undergoes three to five rounds of synchronous mitosis and enters meiotic division (mitotic and meiotic Gcys), while some germ cells are eliminated by cell death. The remaining germ cells enter the diplotene stage of meiosis (Gdip). Oocytes in the diplotene stage undergo the folliculogenesis as they exit from the germinal cradle to the stromal compartment. 
Actually EGFP was only observed in nanos2-expressing single isolated germ cells just after heat induction. But all the germ cells in the germinal cradles became EGFP-positive with time going. These results indicate that nanos2-expressing cells supply all types of germ cells in the germinal cradles.

Next we have checked if the excised DsRed transgene was transmitted to the next generation. Embryos having the excised region were obtained from the transgenic adult medaka even three months after heat-treatment, indicating that nanos2-expressing cells keep supplying the functional eggs. In addition, BrdU incorporation analysis has revealed two populations of the nanos2-expressing cells in terms of cell cycles; fast-dividing (Gsf) and slow-dividing (Gss) germ cells. These results indicate that some populations of nanos2-expressing germ cells contain the germline stem cells. This also demonstrates that the germline stem cell niche exists in the germinal cradle composed of sox $9 b$-expressing cells.

In summary of oogensis from germline stem cells in germinal cradles, we have shown the model in Figure 3C. Fast-dividing (Gsf) and slow-dividing (Gss) germ cells are present among nanos2-expressing single isolated germ cells and both or either of them

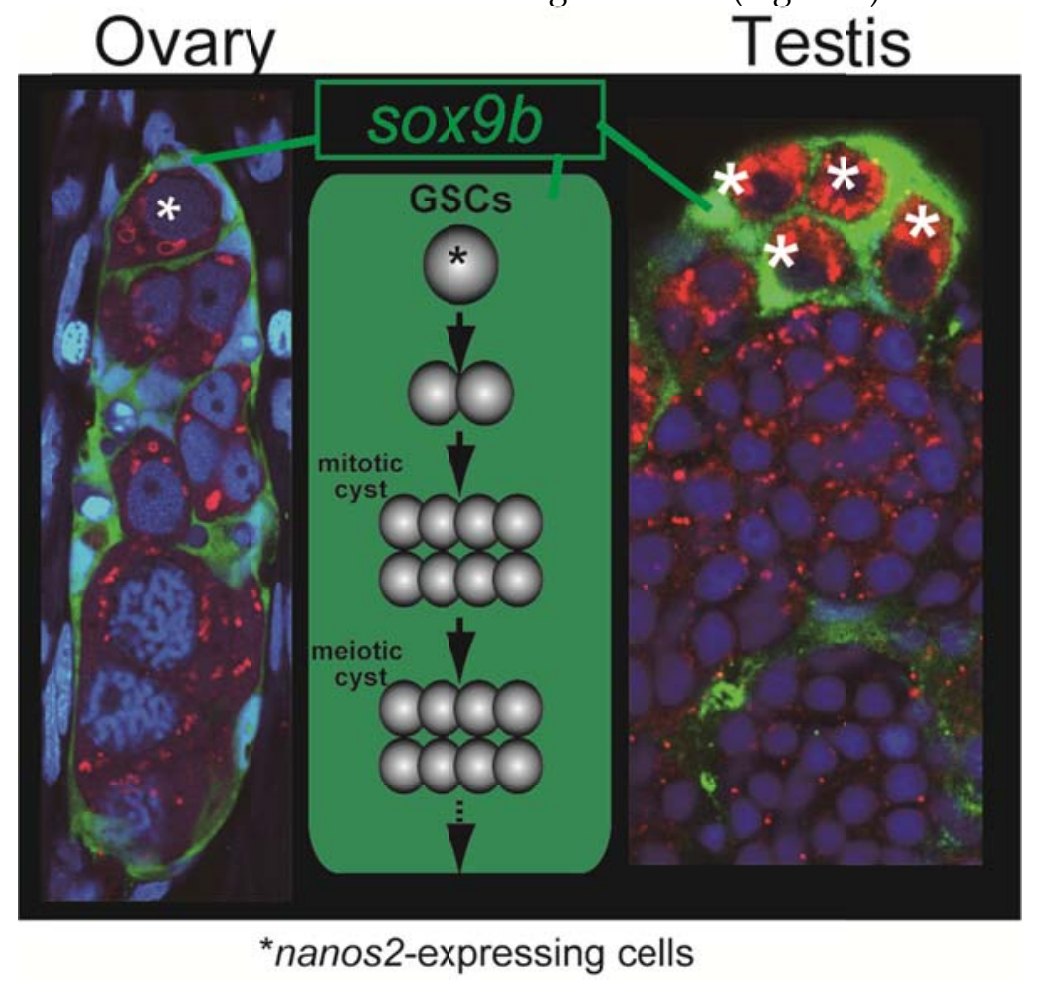

Figure 3. Common structure in ovaries and testes. Does sox $9 b$ have function common in regulating both male and female gremline stem cells? Nanos2-expressing germ cells (asterisks) are surrounded by sox $9 b$-expressing cells one by one in both ovaries and testes. A germinal cradle (left) and a lobule structure (right) are shown. The lobule is a typical unit in the testis. The middle depicts the process and structure common between the germinal cradle and the lobule, which reminds us of the functional unit of oogenesis, called 'germarium' in Drosophila.

function as germline stem cells. The single isolated germ cells undergo the synchronous mitotic and meiotic division to form cyst-forming germ cells. During the cystic division, some germ cells are eliminated by cell death. Similar elimination at around birth is also reported in mammalian ovary [2]. The remaining germ cells enter the diplotene stage of meiosis as ensheathment proceeds by the sox $9 b$-expressing cells one by one in the germinal cradles. Interestingly, the diplotene oocytes are very motile along or within ovarian cords and shuttle back and forth between the germinal cradles. The diplotene oocytes undergo folliculogenesis as they exit to the stromal compartment where enormous oocyte growth and a subsequent maturation event take place. The supporting cells surrounding diplotene oocytes no longer express sox $9 b$ but foxl2 that is often used as a marker of female supporting cells [11].

The oogenetic process in the germinal cradle resembles that in the Drosophila germarium. Germarium contains germline stem cells, cyst-forming germ cells and meiotic germ cells at diplotene stage. The germ cells, as meiotic events proceed, undergo folliculogenesis with somatic supporting cells and exit from germarium (Figure 3).

\section{Testis}




\section{Common structure in ovary and testis and the function of $\operatorname{sox} 9$}

The structure of germinal cradles and the oogenetic events occurring in germinal cradles recall spermatogensis in medaka. Spermatogonia express nanos2 and spermatogenesis proceeds in certain units called lobules that are surrounded by sox $9 b$-exressing cells [16] (Figure 3). This is also reminiscent of mouse spermatogenesis. In mouse testes, nanos2-expressing cells contain the germline stem cells. Spermatogenesis occurs in the tubular structure expressing sox9 [18]. Our previous analysis revealed that sox $9 b$-expressing cells are established before gonadal primordium is present in embryos [19]. These cells form gonadal primordium with another somatic cell expressing $f t z-f 1$ and primordial germ cells. Then, sox $9 b$-expressing cells in the primordium differentiate into either male or female supporting cells (which are subsequently called Sertoli cells in the testis and granulosa cells in the ovary, respectively) [11]. Considering that the sex of medaka can be artificially reversed after sex determination [20] and that female and male supporting cells share a common origin expressing sox $9 b$, it might be interesting to assume that germline stem cells and surrounding sox $9 b$-expressing cells in germinal cradles be sexually indifferent or interchangeable, or maintain sexual bipotentiality.

Another interesting question raised from our results is the function of sox 9 gene in the gonads. Medaka has two orthologues of mammalian sox9 gene, sox $9 a$ and sox $9 b$, but medaka gonads express only sox $9 b$. Therefore sox $9 b$ can be regarded as a functional orthologue of mammalian sox 9 in medaka gonads [21]. Mammalian sox9 is known to have an essential role for Sertoli cell differentiation while sox $9 b$ is expressed in both male and female supporting cells in medaka. In lower vertebrates like medaka, it is intriguing to know that sox $9 b$ has some contribution to male determination.

Recent research in stem cell biology points out another interesting function of a family of the SOX transcription factors. Sox 9 in other tissues and other members of the sox family have been implicated in maintaining cells in a stem cell-like state and inhibiting cell differentiation. For examples, in the central nervous system, Sox1, Sox2, and Sox3 mark neural stem/progenitor cells and prevent their exit from the cell cycle and the induction of neurogenesis [22, 23]. Sox 9 plays a similar role in stem/progenitor cells of the hair bulge, intestinal epithelium, pancreas and neural crest [24-27]. In addition, it has been reported that conditional sox9 knockout testes failed to maintain germ cell [28]. Functional analysis of sox $9 b$ in medaka ovaries may lead to further understanding of some function on germ cells and reproduction.

\section{Conclusions}

We reviewed medaka ovarian germline stem cells and the surrounding unique structure called germinal cradle. Germinal cradles in medaka provide a good model to analyze the oogenesis and the regulation of germline stem cells in vertebrate ovaries. Further studies are required to identify the cells that function as niche in germinal cradles and to reveal the molecular mechanisms regulating germline stem cells.

The germinal cradles also give a deep insight on a variety of sex differentiation process in the gonads of many vertebrates. It is important to examine if similar structures and cells are present in other animals, especially for understanding the relation of a variety of reproductive strategies that many animal show to the cellular and molecular mechanisms that underlie the strategies. Actually, sox 9 expression in both ovaries and testes is reported in rice eels and frogs $[29,30]$, which may indicate that the structure in and the processes of oogenesis shown in medaka are universal among vertebrates.

\section{Acknowledgements}

This work was supported by Grants-in-Aid for Scientific Research on Innovative Areas, (21116509 and 22132001), for Young Scientists (B) (21770072) and for Scientific Research on Priority Areas (B) (21370101).

\section{Conflict of Interests}

The authors have declared that no conflict of interest exists.

\section{References}

1. Nakamura S, Kobayashi K, Nishimura T, et al. Identification of germline stem cells in the ovary of the teleost medaka. Science. 2010; 328: 1561-1563.

2. Pepling ME and Spradling AC. Mouse ovarian germ cell cysts undergo programmed breakdown to form primordial follicles. Dev. Biol. 2001; 234: 339-351.

3. Pepling M.E and Spradling A.C. Female mouse germ cells form synchronously dividing cysts. Development. 1998; 125: 3323-3328.

4. Wallace R.A and Selman K. Ultrastructural aspects of oogenesis and oocyte growth in fish and amphibians. J. Electron Micr. Tech. 1990; 16: 175-201.

5. Tokarz R.R. Oogonial proliferation, oogenesis and folliculogenesis in nonmammalian vertebrates. In: Jones R.E, Editor. The Vertebrate Ovary. New York: Plenum Press. 1978:145-179. 
6. Johnson J, Canning J, Kaneko T, et al. Germline stem cells and follicular renewal in the postnatal mammalian ovary. Nature. 2004; 428: 145-150.

7. Johnson J, Bagley J, Skaznik-Wikiel M, et al. Oocyte generation in adult mammalian ovaries by putative germ cells in bone marrow and peripheral blood. Cell. 2005; 122: 303-315.

8. Eggan K, Jurga S, Gosden R, et al. Ovulated oocytes in adult mice derive from non-circulating germ cells. Nature. 2006; 441: 1109-1114.

9. Nakamura S, Saito D, and Tanaka M. Generation of transgenic medaka using modified bacterial artificial chromosome. Dev. Growth Differ. 2008; 50: 415-419.

10. Tanaka M, Kinoshita M, Kobayashi D, et al. Establishment of medaka (Oryzias latipes) transgenic lines with the expression of green fluorescent protein fluorescence exclusively in germ cells: A useful model to monitor germ cells in a live vertebrate. Proc. Natl. Acad. Sci. U.S.A. 2001; 98: 2544-2549.

11. Nakamura S, Aoki Y, Saito D, et al. Sox9b/sox9a2-EGFP transgenic medaka reveals the morphological reorganization of the gonads and a common precursor of both the female and male supporting cells. Mol. Reprod. Dev. 2008; 75: 472-476.

12. Wagner T, Wirth J, Meyer J, et al. Autosomal sex reversal and campomelic dysplasia are caused by mutations in and around the Sry-related gene Sox9. Cell. 1994; 79: 1111-1120.

13. Kent J, Wheatley S.C, Andrews J.E, et al. A male-specific role for SOX9 in vertebrate sex determination. Development. 1996; 122: 2813-2822.

14. Koopman P, Bullejos M, and Bowles J. Regulation of male sexual development by Sry and Sox9. J. Exp. Zoolog. 2001; 290: 463-474.

15. Vidal V.P.I, Chaboissier M.C, de Rooij D.G, et al. Sox9 induces testis development in XX transgenic mice. Nat. Genet. 2001; 28: 216-217.

16. Aoki Y, Nakamura S, Ishikawa Y, et al. Expression and syntenic analyses of four nanos genes in medaka. Zool. Sci. 2009; 26: 112-118.

17. Kurokawa H, Aoki Y, Nakamura S, et al. Time-lapse analysis reveals different modes of primordial germ cell migration in the medaka Oryzias latipes. Dev. Growth Differ. 2006; 48: 209-221.

18. Sada A, Suzuki A, Suzuki H, et al. The RNA-binding protein NANOS2 is required to maintain murine spermatogonial stem cells. Science. 2009; 325: 1394-1398.

19. Nakamura S, Kobayashi D, Aoki Y, et al. Identification and lineage tracing of two populations of somatic gonadal precursors in medaka embryos. Dev. Biol. 2006; 295: 678-688.

20. Yamamoto T. Artificial induction of functional sex-reversal in genotypic females of the medaka (Oryzias latipes). J. Exp. Zool. 1958; 137: 227-263.

21. Klüver N, Kondo M, Herpin A, et al. Divergent expression patterns of Sox 9 duplicates in teleosts indicate a lineage specific subfunctionalization. Dev. Genes Evol. 2005; 215: 297-305.

22. Bylund M, Andersson E, Novitch B.G, et al. Vertebrate neurogenesis is counteracted by Sox1-3 activity. Nat Neurosci. 2003; 6: 1162-1168.

23. Graham V, Khudyakov J, Ellis P, et al. SOX2 functions to maintain neural progenitor identity. Neuron. 2003; 39: 749-765.

24. Cheung $M$ and Briscoe J. Neural crest development is regulated by the transcription factor Sox9. Development. 2003; 130: 5681-5693.

25. Vidal V.P.I, Chaboissier M.C, Lutzkendorf S, et al. Sox9 is essential for outer root sheath differentiation and the formation of the hair stem cell compartment. Curr. Biol. 2005; 15: 1340-1351.

26. Seymour P.A, Freude K.K, Tran M.N, et al. SOX9 is required for maintenance of the pancreatic progenitor cell pool. Proc. Natl. Acad. Sci. U.S.A. 2007; 104: 1865-1870.

27. Blache $\mathrm{P}$, van de Wetering $\mathrm{M}$, Duluc I, et al. SOX9 is an intestine crypt transcription factor, is regulated by the Wnt pathway, and represses the CDX2 and MUC2 genes. J Cell Biol. 2004; 166: $37-47$.

28. Barrionuevo F, Georg I, Scherthan H, et al. Testis cord differentiation after the sex determination stage is independent of Sox 9 but fails in the combined absence of Sox9 and Sox8. Dev. Biol. 2009; 327: 301-312.

29. Takase M, Noguchi S, and Nakamura M. Two Sox 9 messenger RNA isoforms: isolation of cDNAs and their expression during gonadal development in the frog Rana rugosa. FEBS Lett. 2000; 466: 249-254.

30. Zhou R, Liu L, Guo Y, et al. Similar gene structure of two Sox9a genes and their expression patterns during gonadal differentiation in a teleost fish, rice field eel (Monopterus albus). Mol. Reprod. Dev. 2003; 66: 211-217. 